\title{
Exponential Stabilty for a Timoshenko System with Nonlocal Delay
}

\author{
C. A. S. NONATO ${ }^{1}$, C. A. RAPOSO ${ }^{2 *}$ and H. H. NGUYEN ${ }^{3}$ \\ Received on January 16, 2020 / Accepted on May 27, 2020
}

\begin{abstract}
The purpose of this paper is to study the Timoshenko system with the nonlocal time-delayed condition. The well-posedness is proved by Hille-Yosida theorem. Exploring the dissipative properties of the linear operator associated with the full damped model, we obtain the exponential stability by using Gearhart-Huang-Prüss theorem.
\end{abstract}

Keywords: Timoshenko system, nonlocal time-delayed condition, exponential stability.

\section{INTRODUCTION}

The history of nonlocal problems with integral conditions for partial differential equations is recent and goes back to [4]. In particular, a review of the progress related to the nonlocal models with integral type was given in [3] with many discussions about physical justifications, advantages, and numerical applications. For a nonlocal hyperbolic equation with integral conditions of the 1st kind, we cite [20]. Dissipative properties associated with the Timoshenko system have been studied by several authors by considering the dissipative mechanism of frictional or viscoelastic type. An interesting problem was brought out when the dissipation acts in different ways on the domain. For the case of terms of time-varying delay in the internal feedbacks, the stability result of the Timoshenko system can be found in [10]. On the other hand, for the case of delay and boundary feedback, we can see in [24]. The Timoshenko beam system with delay in the boundary control was studied in [25] where the exponential stabilization result is proved via a test of exact observability of the system. Distributed delay in the boundary control was considered in [13]. Distributive delay in a Timoshenko-type system of thermoelasticity of type III was considered in [8] and then, in [9], the same problem was dealt with constant delay. The Timoshenko system with second sound and the internal distributed delay was investigated in [2].

\footnotetext{
*Correspondent author: Carlos Alberto Raposo - Email:raposo@ufsj.edu.br

${ }^{1}$ Instituto de Matemática e Estatística, Universidade Federal da Bahia, Salvador, BA, Brazil - E-mail: carlos.mat.nonato@hotmail.com https://orcid.org/0000-0002-3672-919X

2 Departamento de Matemática e Estatística, Universidade Federal de São João del-Rei, MG, Brazil - E-mail: raposo@ufsj.edu.br https://orcid.org/0000-0001-8014-7499

${ }^{3}$ LMAP UMR E2S-UPPA CNRS 5142, Bat. IPRA, Avenue de l'Université, 64013 Pau, France - E-mail: huy-hoang.nguyen@univ-pau.fr https://orcid.org/0000-0001-8402-0241
} 
The transmission problem with delay in porous-elasticity was considered in [21]. For a nonlinear Timoshenko system with delay, we cite [5] and reference therein. As far as we know, there is no result for the Timoshenko system with nonlocal delay.

Let $\Omega=(0, L)$ be an interval on $\mathbb{R}$. In this paper, $\varphi=\varphi(x, t)$ describe the small transverse displacement of the beam and $\psi=\psi(x, t)$ the rotation angle of a filament of the beam in $\Omega$, respectively at the time $t$. For a constant $c>0$ and

$$
F, G:(0, c) \rightarrow \mathbb{R}
$$

bounded functions, we define the nonlocal time delayed integral of the 1st kind condition by

$$
\int_{0}^{c} F(s) \varphi_{t}(x, t-s) d s, \int_{0}^{c} G(s) \psi_{t}(x, t-s) d s .
$$

These conditions (1.1) are called nonlocal because the integral is not a pointwise relation, so it provides a problem with distributed delay. Well-posedness and exponential stability for this kind of nonlocal time-delayed for a wave equation were studied in [17,22] by different techniques.

Let $b, k, \alpha, \beta$ be positive constants. The Timoshenko system with frictional damping and nonlocal time-delayed condition is given by

$$
\begin{array}{r}
\rho_{1} \varphi_{t t}-k\left(\varphi_{x}+\psi\right)_{x}+\alpha \varphi_{t}+\int_{0}^{c} F(s) \varphi_{t}(x, t-s) d s=0, \text { in } \Omega \times(0, \infty), \\
\rho_{2} \psi_{t t}-b \psi_{x x}+k\left(\varphi_{x}+\psi\right)+\beta \psi_{t}+\int_{0}^{c} G(s) \psi_{t}(x, t-s) d s=0, \text { in } \Omega \times(0, \infty), \\
\varphi(x, 0)=\varphi_{0}(x) \quad \text { and } \quad \psi(x, 0)=\psi_{0}(x), \quad x \in \Omega, \quad x \in \Omega, \\
\varphi_{t}(x, 0)=\varphi_{1}(x) \quad \text { and } \quad \psi_{t}(x, 0)=\psi_{1}(x), \quad x \in \Omega, s \in(0, c), \\
\varphi_{t}(x,-s)=f_{0}(x,-s), \quad x \in \Omega, s \in(0, c) . \\
\psi_{t}(x,-s)=g_{0}(x,-s), \quad x \in
\end{array}
$$

We consider the Dirichlet boundary conditions as follows

$$
\varphi(0, t)=\varphi(L, t)=0 \quad \text { and } \quad \psi(0, t)=\psi(L, t)=0, \quad t>0 .
$$

Here the initial data

$$
\left(\varphi_{0}(x), \psi_{0}(x)\right) \in H_{0}^{1}(0,1) \times H_{0}^{1}(0,1), \quad\left(\varphi_{1}(x), \psi_{1}(x)\right) \in L^{2}(0,1) \times L^{2}(0,1),
$$

$f_{0}(x,-s), g_{0}(x,-s)$ belong to suitable spaces and

$$
\int_{0}^{c} F(s) d s<\alpha \quad \text { and } \quad \int_{0}^{c} G(s) d s<\beta .
$$

We use the Sobolev spaces with its properties as in Adams [1] and the semigroup theory ( see Pazy [18]). In this paper, we apply the semigroup technique for dissipative systems (see Liu and 
Zheng [14]), that is different from some others in the literature, for example, like as the energy method (see Rivera [23]), the direct method (see Kormonik [11, 12]) and the Nakao's method (see [15]). This manuscript is organized as follows. In Section 2, we deal with the semigroup setting where we prove the well-posedness of the system. In section 3, we show the exponential stability by using the Gearhart-Huang-Prüss theorem, $[6,7,19]$.

\section{SEMIGROUP SETUP}

As in Nicaise and Pignotti [17] we introduce the new variables

$$
\begin{aligned}
& z(x, \rho, t, s)=\varphi_{t}(x, t-\rho s), \quad(x, \rho, t, s) \in Q, \\
& y(x, \rho, t, s)=\psi_{t}(x, t-\rho s), \quad(x, \rho, t, s) \in Q,
\end{aligned}
$$

where $Q=\Omega \times(0,1) \times(0, \infty) \times(0, c)$.

The new variables $z, y$ satisfy

$$
\begin{aligned}
& s z_{t}(x, \rho, t, s)+z_{\rho}(x, \rho, t, s)=0, \\
& s y_{t}(x, \rho, t, s)+y_{\rho}(x, \rho, t, s)=0 .
\end{aligned}
$$

Moreover, using the approach as in [16], the equations

$$
\begin{gathered}
\lambda s z(x, \rho, t, s)+z_{\rho}(x, \rho, t, s)=f, \quad \text { with } \lambda>0, f \in L^{2}(Q), \\
\lambda s y(x, \rho, t, s)+y_{\rho}(x, \rho, t, s)=g, \quad \text { with } \lambda>0, g \in L^{2}(Q)
\end{gathered}
$$

has unique solution

$$
\begin{aligned}
& z(x, \rho, t, s)=z(x, 0, t, s) e^{-\lambda \rho s}+e^{-\lambda \rho s} \int_{0}^{\rho} e^{\lambda \sigma s} f(x, \sigma, t, s) d \sigma \\
& y(x, \rho, t, s)=y(x, 0, t, s) e^{-\lambda \rho s}+e^{-\lambda \rho s} \int_{0}^{\rho} e^{\lambda \sigma s} g(x, \sigma, t, s) d \sigma,
\end{aligned}
$$

respectively. The problem (1.2)-(1.7) is equivalent to

$$
\begin{gathered}
\rho_{1} \varphi_{t t}-k\left(\varphi_{x}+\psi\right)_{x}+\alpha \varphi_{t}+\int_{0}^{c} F(s) z(x, 1, t, s) d s=0,(x, t) \in \Omega \times(0, \infty), \\
\rho_{2} \psi_{t t}-b \psi_{x x}+k\left(\varphi_{x}+\psi\right)+\beta \psi_{t}+\int_{0}^{c} G(s) y(x, 1, t, s) d s=0,(x, t) \in \Omega \times(0, \infty), \\
s z_{t}(x, \rho, t, s)+z_{\rho}(x, \rho, t, s)=0, \quad(x, \rho, t, s) \in Q, \\
s y_{t}(x, \rho, t, s)+y_{\rho}(x, \rho, t, s)=0, \quad(x, \rho, t, s) \in Q, \\
\varphi(x, 0)=\varphi_{0}(x) \quad \text { and } \quad \psi(x, 0)=\psi_{0}(x), \quad x \in \Omega, \\
\varphi_{t}(x, 0)=\varphi_{1}(x) \quad \text { and } \quad \psi_{t}(x, 0)=\psi_{1}(x), \quad x \in \Omega, \\
z(x, \rho, 0, s)=f_{0}(x,-\rho s), \quad(x, \rho, s) \in \Omega \times(0,1) \times(0, c), \\
\varphi(x, \rho, 0, s)=g_{0}(x,-\rho s), \quad(x, \rho, s) \in \Omega \times(0,1) \times(0, c),
\end{gathered}
$$


with the Dirichlet boundary condition (1.8) and $z(x, \rho, t, s)=y(x, \rho, t, s)=0$ on the boundary $x=0, L$.

Defining $U=(\varphi, \psi, u, v, z, y)^{T}, u=\varphi_{t}$ and $v=\psi_{t}$, we formally get that $U$ satisfies the Cauchy problem

$$
\begin{gathered}
U_{t}=\mathscr{A} U, \quad t>0, \\
U(0)=U_{0}=\left(\varphi_{0}, \psi_{0}, \varphi_{1}, \psi_{1}, f_{0}, g_{0}\right)^{T},
\end{gathered}
$$

where the operator $\mathscr{A}$ is defined by

$$
\mathscr{A} U=\left[\begin{array}{c}
u \\
v \\
\frac{1}{\rho_{1}}\left[k\left(\varphi_{x}+\psi\right)_{x}-\alpha u-\int_{0}^{c} F(s) z(x, 1, t, s) d s\right] \\
\frac{1}{\rho_{2}}\left[b \psi_{x x}-k\left(\varphi_{x}+\psi\right)-\beta v-\int_{0}^{c} G(s) y(x, 1, t, s) d s\right] \\
-s^{-1} z_{\rho}(x, \rho, t, s) \\
-s^{-1} y_{\rho}(x, \rho, t, s)
\end{array}\right]
$$

We introduce the energy space

$$
\mathscr{H}=H_{0}^{1}(\Omega)^{2} \times L^{2}(\Omega)^{2} \times L^{2}(\Omega \times(0,1) \times(0, c))^{2}
$$

equipped with the inner product

$$
\begin{aligned}
\langle U, \bar{U}\rangle_{\mathscr{H}}= & \int_{\Omega}\left[k\left(\varphi_{x}+\psi\right)\left(\bar{\varphi}_{x}+\bar{\psi}\right)+\rho_{1} u \bar{u}+\rho_{2} v \bar{v}+b \psi_{x} \bar{\psi}_{x}\right] d x \\
& +\int_{\Omega} \int_{0}^{1} \int_{0}^{c} s F(s) z \bar{z} d s d \rho d x+\int_{\Omega} \int_{0}^{1} \int_{0}^{c} s G(s) y \bar{y} d s d \rho d x
\end{aligned}
$$

for $U=(\varphi, \psi, u, v, z, y)^{T}$ and $\bar{U}=(\bar{\varphi}, \bar{\psi}, \bar{u}, \bar{v}, \bar{z}, \bar{y})^{T}$.

The domain of $\mathscr{A}$ is defined by

$$
D(\mathscr{A})=\left[H^{2}(\Omega) \cap H_{0}^{1}(\Omega)\right]^{2} \times H_{0}^{1}(\Omega)^{2} \times L^{2}\left(\Omega \times(0,1) ; H^{1}(\Omega)\right)^{2} .
$$

Clearly, $D(\mathscr{A})$ is dense in $\mathscr{H}$ and independent of time $t>0$. Next, we will prove that the operator $\mathscr{A}$ is dissipative.

Lemma 2.1. For $U=(\varphi, \psi, u, v, z, y) \in D(\mathscr{A})$. Assume that

$$
\int_{0}^{c} F(s) d s<\alpha \quad \text { and } \quad \int_{0}^{c} G(s) d s<\beta
$$

then we have

$$
\begin{aligned}
\langle\mathscr{A} U, U\rangle_{\mathscr{H}} \leq & -\left(\alpha-\int_{0}^{c} F(s) d s\right) \int_{\Omega}|u|^{2} d x \\
& -\left(\beta-\int_{0}^{c} G(s) d s\right) \int_{\Omega}|v|^{2} d x \\
\leq & 0 .
\end{aligned}
$$




\section{Proof.}

$$
\begin{aligned}
\langle\mathscr{A} U, U\rangle_{\mathscr{H}}= & k \int_{\Omega}\left(u_{x}+v\right)\left(\varphi_{x}+\psi\right) d x \\
& +\int_{\Omega}\left[k\left(\varphi_{x}+\psi\right)_{x}-\alpha u-\int_{0}^{c} F(s) z(x, 1, t, s) d s\right] u d x \\
& +\int_{\Omega}\left[b \psi_{x x}-k\left(\varphi_{x}+\psi\right)-\beta v-\int_{0}^{c} G(s) y(x, 1, t, s) d s\right] v d x \\
& +b \int_{\Omega} v_{x} \psi_{x} d x-\int_{\Omega} \int_{0}^{1} \int_{0}^{c} F(s) z_{\rho}(x, \rho, t, s) z(x, \rho, t, s) d s d \rho d x \\
& -\int_{\Omega} \int_{0}^{1} \int_{0}^{c} G(s) y_{\rho}(x, \rho, t, s) y(x, \rho, t, s) d s d \rho d x .
\end{aligned}
$$

Integrating by parts on $\Omega$,

$$
\begin{aligned}
\langle\mathscr{A} U, U\rangle_{\mathscr{H}}= & -\alpha \int_{\Omega}|u|^{2} d x-\int_{\Omega} \int_{0}^{c} F(s) z(x, 1, t, s) u d s d x \\
& -\beta \int_{\Omega}|v|^{2} d x-\int_{\Omega} \int_{0}^{c} G(s) y(x, 1, t, s) v d s d x \\
& -\int_{\Omega} \int_{0}^{1} \int_{0}^{c} F(s) z_{\rho}(x, \rho, t, s) z(x, \rho, t, s) d s d \rho d x \\
& -\int_{\Omega} \int_{0}^{1} \int_{0}^{c} G(s) y_{\rho}(x, \rho, t, s) y(x, \rho, t, s) d s d \rho d x .
\end{aligned}
$$

Taking into account $z(x, 0, t, s)=\varphi_{t}(x, t)=u, y(x, 0, t, s)=\psi_{t}(x, t)=v$ we have

$$
\begin{aligned}
& \int_{\Omega} \int_{0}^{1} \int_{0}^{c} F(s) z_{\rho}(x, \rho, t, s) z(x, \rho, t, s) d s d \rho d x \\
& =\int_{\Omega} \int_{0}^{c} \int_{0}^{1} F(s) \frac{1}{2} \frac{\partial}{\partial \rho}|z(x, \rho, t, s)|^{2} d \rho d s d x \\
& =\frac{1}{2} \int_{\Omega} \int_{0}^{c} F(s)|z(x, 1, t, s)|^{2} d s d x-\frac{1}{2} \int_{0}^{c} F(s) d s \int_{\Omega}|u|^{2} d x
\end{aligned}
$$

and

$$
\begin{aligned}
& \int_{\Omega} \int_{0}^{1} \int_{0}^{c} G(s) y_{\rho}(x, \rho, t, s) y(x, \rho, t, s) d s d \rho d x \\
& =\frac{1}{2} \int_{\Omega} \int_{0}^{c} G(s)|y(x, 1, t, s)|^{2} d s d x-\frac{1}{2} \int_{0}^{c} G(s) d s \int_{\Omega}|v|^{2} d x .
\end{aligned}
$$

Inserting (2.17) and (2.18) into (2.16), apply Young's inequality and simplifying the terms, we obtain

$$
\langle\mathscr{A} U, U\rangle_{\mathscr{H}} \leq-\left(\alpha-\int_{0}^{c} F(s) d s\right) \int_{\Omega}|u|^{2} d x-\left(\beta-\int_{0}^{c} G(s) d s\right) \int_{\Omega}|v|^{2} d x .
$$

Finally, by the assumption (2.14) we conclude the proof. 
The well-posedness of (2.5)-(2.12) is ensured by the following theorem.

Teorema 2.1. For $U_{0} \in \mathscr{H}$, there exists a unique weak solution $U$ of (2.13) satisfying

$$
U \in C((0, \infty) ; \mathscr{H}) .
$$

Moreover, if $U_{0} \in D(\mathscr{A})$, then

$$
U \in C((0, \infty) ; D(\mathscr{A})) \cap C^{1}((0, \infty) ; \mathscr{H}) .
$$

Proof. We will use the Hille-Yosida theorem. Since $\mathscr{A}$ is dissipative and $D(\mathscr{A})$ is dense in $\mathscr{H}$, it is sufficient to show that $\mathscr{A}$ is maximal; that is, $I-\mathscr{A}$ is surjective. Given $H=\left(h_{1}, h_{2}, \ldots, h_{6}\right) \in$ $\mathscr{H}$, we must show that there exists $U=(\varphi, \psi, u, v, z, y) \in D(\mathscr{A})$ satisfying $(I-\mathscr{A}) U=H$ which is equivalent to

$$
\begin{gathered}
\varphi-u=h_{1}, \\
\psi-v=h_{2}, \\
\rho_{1} u-k\left(\varphi_{x}+\psi\right)_{x}+\alpha u+\int_{0}^{c} F(s) z(x, 1, t, s) d s=\rho_{1} h_{3}, \\
\rho_{2} v-b \psi_{x x}+k\left(\varphi_{x}+\psi\right)+\beta v+\int_{0}^{c} G(s) y(x, 1, t, s) d s=\rho_{2} h_{4}, \\
s z(x, \rho, t, s)+z_{\rho}(x, \rho, t, s)=s h_{5}, \\
s y(x, \rho, t, s)+y_{\rho}(x, \rho, t, s)=s h_{6} .
\end{gathered}
$$

Suppose that we have found $\varphi$ and $\psi$ with the appropriated regularity. Therefore, (2.21) and (2.22) give

$$
\begin{aligned}
& u=\varphi-h_{1}, \\
& v=\psi-h_{2} .
\end{aligned}
$$

It is clear that $u, v \in H_{0}^{1}(\Omega)$.

From (2.1),(2.3) it follows that equation (2.25) has a unique solution given by

$$
z(x, \rho, s)=u(x) e^{-\rho s}+s e^{-\rho s} \int_{0}^{\rho} e^{\sigma s} h_{5}(x, \sigma, s) d \sigma
$$

and from (2.2),(2.4) it follows that equation (2.26) has a unique solution given by

$$
y(x, \rho, s)=v(x) e^{-\rho s}+s e^{-\rho s} \int_{0}^{\rho} e^{\sigma s} h_{6}(x, \sigma, s) d \sigma .
$$

So, from (2.27) and (2.28),

$$
\begin{aligned}
& z(x, \rho, s)=\varphi(x) e^{-\rho s}-h_{1}(x) e^{-\rho s}+s e^{-\rho s} \int_{0}^{\rho} e^{\sigma s} h_{5}(x, \sigma, s) d \sigma \\
& y(x, \rho, s)=\psi(x) e^{-\rho s}-h_{2}(x) e^{-\rho s}+s e^{-\rho s} \int_{0}^{\rho} e^{\sigma s} h_{6}(x, \sigma, s) d \sigma
\end{aligned}
$$


and, in particular,

$$
\begin{aligned}
& z(x, 1, s)=\varphi(x) e^{-s}+z_{0}(x, s), \\
& y(x, 1, s)=\psi(x) e^{-s}+y_{0}(x, s),
\end{aligned}
$$

where $z_{0}(x, s), y_{0}(x, s) \in L^{2}(\Omega \times(0, c))$ defined by

$$
\begin{aligned}
& z_{0}(x, s)=-h_{1}(x) e^{-s}+s e^{-s} \int_{0}^{1} e^{\sigma s} h_{5}(x, \sigma, s) d \sigma \\
& y_{0}(x, s)=-h_{2}(x) e^{-s}+s e^{-s} \int_{0}^{1} e^{\sigma s} h_{6}(x, \sigma, s) d \sigma .
\end{aligned}
$$

By (2.23), (2.24), (2.27) and (2.28), we see that the functions $\varphi$ and $\psi$ satisfy the following system

$$
\left\{\begin{array}{l}
\lambda \varphi-k\left(\varphi_{x}+\psi\right)_{x}=g_{1}, \\
\eta \psi-b \psi_{x x}+k\left(\varphi_{x}+\psi\right)=g_{2}
\end{array}\right.
$$

where

$$
\begin{gathered}
\lambda=\rho_{1}+\alpha+\int_{0}^{c} e^{-s} F(s) d s, \quad \eta=\rho_{2}+\beta+\int_{0}^{c} e^{-s} G(s) d s, \\
g_{1}=\rho_{1} h_{1}+\alpha h_{1}+\rho_{1} h_{3}-\int_{0}^{c} F(s) z_{0}(x, s) d s, \\
g_{2}=\rho_{2} h_{2}+\beta h_{2}+\rho_{2} h_{4}-\int_{0}^{c} G(s) y_{0}(x, s) d s .
\end{gathered}
$$

Solving the system (2.29) is equivalent to finding $(\varphi, \psi) \in\left[H^{2}(\Omega) \cap H_{0}^{1}(\Omega)\right]^{2}$ such that

$$
\left\{\begin{array}{l}
\lambda \int_{\Omega} \varphi \tilde{\varphi} d x+k \int_{\Omega}\left(\varphi_{x}+\psi\right) \tilde{\varphi}_{x} d x=\int_{\Omega} g_{1} \tilde{\varphi} d x \\
\eta \int_{\Omega} \psi \tilde{\psi} d x+b \int_{\Omega} \psi_{x} \tilde{\psi}_{x} d x+k \int_{\Omega}\left(\varphi_{x}+\psi\right) \tilde{\psi} d x=\int_{\Omega} g_{2} \tilde{\psi} d x,
\end{array}\right.
$$

for all $(\tilde{\varphi}, \tilde{\psi}) \in H_{0}^{1}(\Omega) \times H_{0}^{1}(\Omega)$.

Now, we observe that solving the system (2.30) is equivalent to solve the problem

$$
\Upsilon((\varphi, \psi),(\tilde{\varphi}, \tilde{\psi}))=L(\tilde{\varphi}, \tilde{\psi})
$$

where the bilinear form

$$
\Upsilon:\left[H_{0}^{1}(\Omega) \times H_{0}^{1}(\Omega)\right]^{2} \rightarrow(0, \infty)
$$

and the linear form

$$
L: H_{0}^{1}(\Omega) \times H_{0}^{1}(\Omega) \rightarrow(0, \infty)
$$

are defined by

$$
\begin{aligned}
\Upsilon((\varphi, \psi),(\tilde{\varphi}, \tilde{\psi}))= & \lambda \int_{\Omega} \varphi \tilde{\varphi} d x+k \int_{\Omega}\left(\varphi_{x}+\psi\right)\left(\tilde{\varphi}_{x}+\tilde{\psi}\right) d x \\
& +\eta \int_{\Omega} \psi \tilde{\psi} d x+b \int_{\Omega} \psi_{x} \tilde{\psi}_{x} d x
\end{aligned}
$$


and

$$
L(\tilde{\varphi}, \tilde{\psi})=\int_{\Omega} g_{1} \tilde{\varphi} d x+\int_{\Omega} g_{2} \tilde{\psi} d x
$$

It is easy to verify that $\Upsilon$ is continuous and coercive, and $L$ is continuous. So applying the LaxMilgram theorem, we deduce that for all $(\tilde{\varphi}, \tilde{\psi}) \in H_{0}^{1}(\Omega) \times H_{0}^{1}(\Omega)$ the problem (2.31) admits a unique solution

$$
(\varphi, \psi) \in H_{0}^{1}(\Omega) \times H_{0}^{1}(\Omega) .
$$

Applying the classical elliptic regularity, it follows from (2.30) that

$$
(\varphi, \psi) \in H^{2}(\Omega) \times H^{2}(\Omega) .
$$

Therefore, the operator $I-\mathscr{A}$ is surjective. As consequence of the Hille-Yosida theorem [14, Theorem 1.2.2, page 3], we have that $\mathscr{A}$ generates a $C_{0}$-semigroup of contractions $S(t)=e^{t \mathscr{A}}$ on $\mathscr{H}$. From semigroup theory, $U(t)=e^{t \mathscr{A}} U_{0}$ is the unique solution of (2.13) satisfying (2.19) and (2.20). The proof is complete.

\section{EXPONENTIAL STABILITY}

The necessary and sufficient conditions for the exponential stability of the $C_{0}$-semigroup of contractions on a Hilbert space were obtained by Gearhart [6] and Huang [7] independently, see also Pruss [19]. We will use the following result due to Gearhart.

Teorema 3.2. Let $\rho(\mathscr{A})$ be the resolvent set of the operator $\mathscr{A}$ and $S(t)=e^{t \mathscr{A}}$ be the $C_{0^{-}}$ semigroup of contractions generated by $\mathscr{A}$. Then, $S(t)$ is exponentially stable if and only if

$$
\begin{aligned}
& i \mathbb{R}=\{i \zeta: \zeta \in \mathbb{R}\} \subset \rho(\mathscr{A}), \\
& \limsup _{|\zeta| \rightarrow \infty}\left\|(i \zeta I-\mathscr{A})^{-1}\right\|<\infty .
\end{aligned}
$$

The main result of this manuscript is the following theorem.

Teorema 3.3. The semigroup $S(t)=e^{t \mathscr{A}}$ generated by $\mathscr{A}$ is exponentially stable.

Proof. It is sufficient to verify (3.1) and (3.2). If (3.1) is not true, it means that there is a $\zeta \in \mathbb{R}$ such that $\zeta \neq 0, i \zeta$ is in the spectrum de $\mathscr{A}$. From the compact immersion of $D(\mathscr{A})$ in $\mathscr{H}$, by spectral theory, there is a vector function

$$
U=(\varphi, \psi, u, v, z, y) \in D(\mathscr{A}), \text { with }\|U\|_{\mathscr{H}}=1,
$$


such that $\mathscr{A} U=i \zeta U$, which is equivalent to

$$
\begin{gathered}
i \zeta \varphi-u=0, \\
i \zeta \psi-v=0, \\
i \zeta u-\frac{1}{\rho_{1}}\left[k\left(\varphi_{x}+\psi\right)_{x}-\alpha u-\int_{0}^{c} F(s) z(x, 1, t, s) d s\right]=0, \\
i \zeta v-\frac{1}{\rho_{2}}\left[b \psi_{x x}-k\left(\varphi_{x}+\psi\right)-\beta v-\int_{0}^{c} G(s) y(x, 1, t, s) d s\right]=0, \\
i \zeta s z(x, \rho, t, s)+z_{\rho}(x, \rho, t, s)=0, \\
i \zeta s y(x, \rho, t, s)+y_{\rho}(x, \rho, t, s)=0 .
\end{gathered}
$$

Using (3.3) we obtain $u_{x}=i \zeta \varphi_{x}$. Multiplying by $u_{x}$, integrating on $\Omega$ and using Young's inequality we have

$$
\int_{\Omega}\left|u_{x}\right|^{2} d x=i \zeta \int_{\Omega} \varphi_{x} u_{x} d x \leq-\frac{1}{2} \zeta^{2} \int_{\Omega}\left|\varphi_{x}\right|^{2} d x+\frac{1}{2} \int_{\Omega}\left|u_{x}\right|^{2} d x
$$

from where it follows that

$$
\frac{1}{2} \zeta^{2} \int_{\Omega}\left|\varphi_{x}\right|^{2} d x+\frac{1}{2} \int_{\Omega}\left|u_{x}\right|^{2} d x \leq 0 .
$$

Applying Poincaré's inequality in (3.9) we obtain $\varphi=u=0$ a.e. in $L^{2}(\Omega)$. Note that (2.3) gives us $z=u e^{-i \zeta \rho s}$ as the unique solution of (3.7), which implies $z=0$ a.e. in $L^{2}(\Omega \times(0,1) \times(0, c))$. Similarly, it is proved that $\psi=v=0$ a.e. in $L^{2}(\Omega)$ and $y=0$ a.e. in $L^{2}(\Omega \times(0,1) \times(0, c))$. But $\varphi=\psi=u=v=z=y=0$ is a contradiction with $\|U\|_{\mathscr{H}}=1$ and then (3.1) holds.

To prove (3.2) we use contradiction argument again. If (3.2) is not true, there exists a real sequence $\zeta_{n}$, with $\zeta_{n} \rightarrow \infty$ and a sequence of vector functions $V_{n} \in \mathscr{H}$ that satisfies

$$
\frac{\left\|\left(\lambda_{n} I-\mathscr{A}\right)^{-1} V_{n}\right\|_{\mathscr{H}}}{\left\|V_{n}\right\|_{\mathscr{H}}} \geq n, \quad \text { where } \lambda_{n}=i \zeta_{n} .
$$

Hence

$$
\left\|\left(\lambda_{n} I-\mathscr{A}\right)^{-1} V_{n}\right\|_{\mathscr{H}} \geq n\left\|V_{n}\right\|_{\mathscr{H}} .
$$

Since $\lambda_{n} \in \rho(\mathscr{A})$ it follows that there exists a unique sequence

$$
U_{n}=\left(\varphi_{n}, \psi_{n}, u_{n}, v_{n}, z_{n}, y_{n}\right)^{T} \in D(\mathscr{A}),
$$

with unit norm in $\mathscr{H}$ such that

$$
\left(\lambda_{n} I-\mathscr{A}\right)^{-1} V_{n}=U_{n}
$$

Denoting $\xi_{n}=\lambda_{n} U_{n}-\mathscr{A} U_{n}$ we have from (3.10) that

$$
\left\|\xi_{n}\right\|_{\mathscr{H}} \leq \frac{1}{n}
$$


and then $\xi_{n} \rightarrow 0$ strongly in $\mathscr{H}$ as $n \rightarrow \infty$.

Taking the inner product of $\xi_{n}$ with $U_{n}$ we have

$$
\lambda_{n}\left\|U_{n}\right\|_{\mathscr{H}}^{2}-\left\langle\mathscr{A} U_{n}, U_{n}\right\rangle_{\mathscr{H}}=\left\langle\xi_{n}, U_{n}\right\rangle_{\mathscr{H}}
$$

Using Lemma 2.1, follows that

$$
\begin{aligned}
& \lambda_{n}\left\|U_{n}\right\|_{\mathscr{H}}^{2}+\left(\alpha-\int_{0}^{c} F(s) d s\right) \int_{\Omega}\left|u_{n}\right|^{2} d x+\left(\beta-\int_{0}^{c} G(s) d s\right) \int_{\Omega}\left|v_{n}\right|^{2} d x \\
& \leq\left\langle\xi_{n}, U_{n}\right\rangle_{\mathscr{H}}
\end{aligned}
$$

and taking the real part we have

$$
\left(\alpha-\int_{0}^{c} F(s) d s\right) \int_{\Omega}\left|u_{n}\right|^{2} d x+\left(\beta-\int_{0}^{c} G(s) d s\right) \int_{\Omega}\left|v_{n}\right|^{2} d x \leq \operatorname{Re}\left\langle\xi_{n}, U_{n}\right\rangle_{\mathscr{H}} .
$$

As $U_{n}$ is bounded and $\xi_{n} \rightarrow 0$ we obtain

$$
u_{n} \rightarrow 0 \quad \text { and } \quad v_{n} \rightarrow 0 \quad \text { as } n \rightarrow \infty
$$

Now, for $\xi_{n}=\left(\xi_{n}^{1}, \xi_{n}^{2}, \ldots, \xi_{n}^{6}\right), \xi_{n}=\lambda_{n} U_{n}-\mathscr{A} U_{n}$ is equivalent to

$$
\begin{gathered}
i \zeta_{n} \varphi_{n}-u_{n}=\xi_{n}^{1}, \\
i \zeta_{n} \psi_{n}-v_{n}=\xi_{n}^{2} \\
i \zeta_{n} u_{n}-\frac{1}{\rho_{1}}\left[k\left(\varphi_{x, n}+\psi_{n}\right)_{x}-\alpha u_{n}-\int_{0}^{c} F(s) z_{n}(x, 1, t, s) d s\right]=\xi_{n}^{3}, \\
i \zeta_{n} v_{n}-\frac{1}{\rho_{2}}\left[b \psi_{x x, n}-k\left(\varphi_{x, n}+\psi_{n}\right)-\beta v_{n}-\int_{0}^{c} G(s) y_{n}(x, 1, t, s) d s\right]=\xi_{n}^{4}, \\
i \zeta_{n} s z_{n}(x, \rho, t, s)+z_{\rho, n}(x, \rho, t, s)=s \xi_{n}^{5}, \\
i \zeta_{n} s y_{n}(x, \rho, t, s)+y_{\rho, n}(x, \rho, t, s)=s \xi_{n}^{6},
\end{gathered}
$$

where

$$
\xi_{n}^{j} \rightarrow 0
$$

for $j=1, \ldots, 6$.

From (3.11), (3.12), (3.13) and (3.18), we obtain that

$$
\varphi_{n} \rightarrow 0 \quad \text { and } \quad \psi_{n} \rightarrow 0 \quad \text { as } n \rightarrow \infty .
$$

By (2.1) and (2.3), we have that

$$
z_{n}(x, \rho, s)=u_{n}(x) e^{-i \zeta_{n} \rho s}+s e^{-i \zeta_{n} \rho s} \int_{0}^{\rho} e^{i \zeta_{n} \sigma s} \xi_{n}^{5}(x, \sigma, s) d \sigma
$$


is the unique solution of (3.16). Using the Euler formula for complex numbers in (3.20) we obtain

$$
\begin{aligned}
z_{n}(x, \rho, s)= & u_{n}(x)\left[\cos \left(\zeta_{n} \rho s\right)-i \sin \left(\zeta_{n} \rho s\right)\right] \\
& +s\left[\cos \left(\zeta_{n} \rho s\right)-i \sin \left(\zeta_{n} \rho s\right)\right] \int_{0}^{\rho}\left[\cos \left(\zeta_{n} \sigma s\right)+i \sin \left(\zeta_{n} \sigma s\right)\right] \xi_{n}^{5}(x, \sigma, s) d \sigma
\end{aligned}
$$

Since $\cos \left(\zeta_{n} \rho s\right)-i \sin \left(\zeta_{n} \rho s\right)$ and $\cos \left(\zeta_{n} \sigma s\right)+i \sin \left(\zeta_{n} \sigma s\right)$ limited and by (3.11), (3.18) deducing that

$$
z_{n}(x, \rho, t) \rightarrow 0 \quad \text { as } n \rightarrow \infty
$$

Analogously, we conclude that

$$
y_{n}(x, \rho, t) \rightarrow 0 \quad \text { as } n \rightarrow \infty .
$$

Finally, (3.11), (3.19), (3.21) and (3.22) give us a contradiction with $\left\|U_{n}\right\|_{\mathscr{H}}=1$. The proof is complete.

RESUMO. O objetivo deste artigo é estudar o sistema de Timoshenko com uma condição de retardo de tempo não local. A boa colocação é provada através do teorema de HilleYosida. Explorando as propriedades dissipativas do operador linear associado ao modelo totalmente amortecido, obtemos a estabilidade exponencial usando o Teorema de GearhartHuang-Prüss.

Palavras-chave: sistema de Timoshenko, condição de retardo não local, estabilidade exponencial.

\section{REFERENCES}

[1] R. A. Adams. Sobolev Spaces. Academic Press, New York 1975.

[2] T. A. Apalara. Well-posedness and exponential stability for a linear damped Timoshenko system with second sound and internal distributed delay. Electron. J. Differential Equations, 254 (2014), 1-15.

[3] Z. P. Bažant \& M. Jirásek. Nonlocal Integral Formulation of Plasticity And Damage: Survey of Progress. J. Eng. Mech., 128 (2002), 1119-1149.

[4] J. R. Cannon. The solution of heat equation subject to the specification of energy. Quart. Appl. Math., 21 (1963), 155-160.

[5] B. Feng \& M. L. Pelicer. Global existence and exponential stability for a nonlinear Timoshenko system with delay. Bound. Value Probl., 26 (2015), https://doi.org/10.1186/s13661015-0468-4.

[6] L. Gearhart. Spectral theory for contraction semigroups on Hilbert spaces. Trans. Amer. Math. Soc., 236 (1978), 385-394. 
[7] F. Huang. Characteristic conditions for exponential stability of linear dynamical systems in Hilbert spaces. Ann. Diff. Eqns., 1 (1985), 45-53.

[8] M. Kafini, S. A. Messaoudi \& M. I. Mustafa. Energy decay result in a Timoshenko-type system of thermoelasticity of type III with distributive delay. J. Math. Phys., 54 (2013), 101503.

[9] M. Kafini, S. A. Messaoudi \& M. I. Mustafa. Energy decay rates for a Timoshenko-type system of thermoelasticity of type III with constant delay. Appl. Anal., 93 (2014), 12011216.

[10] M. Kirane, B. Said-Houari \& M. N. Anwar. Stability result for the Timoshenko system with a time-varying delay term in the internal feedbacks. Commun. Pur. Appl. Anal. , 10 (2011), 667-686.

[11] V. Komornik. Exact controllability and stabilization. The multiplier method. RAM: Research in Applied Mathematics, John Wiley \& Sons, Paris, 1994.

[12] V. Kormonik \& E. Zuazua. A direct method for the boundary stabilization of the wave equation. J. Math. Pures Appl., 69 (1990), 33-54.

[13] X. F. Liu \& G. Q. Xu. Exponential Stabilization for Timoshenko Beam with Distributed Delay in the Boundary Control. Abstr. Appl. Anal.. 2013 (2013), 726794.

[14] Z. Liu \& S. Zheng. Semigroups Associated with Dissipative Systems. Chapman \& Hall 1999.

[15] M. Nakao. On the decay of solutions of some nonlinear dissipative wave equations. Math. Z. Berlin., 193 (1986), 227-234 .

[16] S. Nicaise \& C. Pignotti. Stability and instability results of the wave equation with a delay term in the boundary or internal feedbacks. SIAM J. Control Optim., 45 (2006), 1561-1585.

[17] S. Nicaise \& C. Pignotti. Stabilization of the wave equation with boundary or internal distributed delay. Differential Integral Equations, 21 (2008), 935-958.

[18] A. Pazy. Semigroups of Linear Operators and Applications to Partial Differential Equations. Springer- Verlag, New York 1983.

[19] J. Pruss. On the spectrum of $C_{0}$-semigroups. Trans. Amer. Math. Soc. 284 (2) (1984), 847857.

[20] L. S. Pul'kina. A nonlocal problem for a hyperbolic equation with integral conditions of the 1 st kind with time-dependent kernels. Izv. Vyssh. Uchebn. Zaved. Mat., 10 (2012), 32-44.

[21] C. A. Raposo, T. A. Apalara \& J. O. Ribeiro. Analyticity to transmission problem with delay in porous-elasticity. J. Math. Anal. Appl., 46 (2018), 819-834. 
[22] C. A. Raposo, H. Nguyen, J. O. Ribeiro \& V. B. Oliveira. Well-posedness and exponential stability for a wave equation with nonlocal time-delay condition. Electron. J. Differential Equations, 279 (2017), 1-11.

[23] J.E.M. Rivera. Energy decay rates in linear thermoelasticity. Funkcial EKVAC, 35 (1992), 9-30.

[24] B. Said-Houari \& A. Soufyane. Stability result of the Timoshenko system with delay and boundary feedback. IMA J. Math. Control Inf., 6 (2012), 1-16.

[25] G. Xu \& H. Wang. Stabilisation of Timoshenko beam system with delay in the boundary control. Int. J. Control., 86 (2013), 1165-1178. 